\title{
Preliminary screening and identification of differentially expressed metastasis-related ncRNAs in ovarian cancer
}

\author{
YU YANG $^{1}$, HUI ZHANG ${ }^{1}$, YAJIAO XIE ${ }^{1}$, SHUFEN ZHANG $^{2}$, JUNYOU ZHU ${ }^{2}$, \\ GANG YIN ${ }^{3}$, GUANG SHU ${ }^{3}$ and YU ZHANG ${ }^{2}$
${ }^{1}$ School of Resources Processing and Bioengineering, Central South University, Changsha, Hunan 410006; ${ }^{2}$ Department of Obstetrics and Gynecology, Xiangya Hospital, Central South University, Changsha, Hunan 410008; ${ }^{3}$ Xiangya Hospital, Central South University, Changsha, Hunan 410013, P.R. China

Received July 3, 2017; Accepted October 19, 2017

DOI: $10.3892 / \mathrm{ol} .2017 .7338$

\begin{abstract}
Ovarian cancer (OC) is an aggressive disease with few valuable biomarkers and effective therapies. In this study, we aimed to elucidate biomarkers associated with OC metastasis into the omentum. We performed comprehensive screening of non-coding RNAs (ncRNAs) between matched primary $\mathrm{OC}$ and omental metastasis using the Agilent human lncRNA Array V3.0 microarray. Reverse transcription quantitative polymerase chain reaction (RT-qPCR) was used to validate the microarray results at the mRNA level. Microarray revealed 235 ncRNAs changes, and we validated the top four differential changed genes in an additional 27 paired samples with RT-qPCR. We found that myocardial infarction associated transcript (MIAT) expression increased in the omentum tissue, while small nucleolar RNA, C/D Box 114 cluster (SNORD114) family members SNORD114-10, SNORD114-2 and SNORD114-11 were downregulated when compared with OC tissue. However, there is no significant difference in SNORD114-2 and SNORD114-11 levels. We thus infer that differential expression of MIAT and SNORD114-10 could play an important role during OC metastasis. These ncRNAs might be useful as pre-diagnostic biomarkers at the early stage of cancer metastasis.
\end{abstract}

\section{Introduction}

Epithelial ovarian cancer (EOC) is the most aggressive malignant gynecological disease, and the 5-year survival rate of patients less than $30 \%$ (1). It is estimated that 22,280 new diagnoses and 14,240 deaths from this neoplasm were reported

Correspondence to: Dr Yu Zhang, Department of Obstetrics and Gynecology, Xiangya Hospital, Central South University, 87 Xiangya Road, Changsha, Hunan 410008, P.R. China

E-mail: xyzhangyu@csu.edu.cn

Key words: ovarian metastasis, omentum, long non-coding RNA, myocardial infarction associated transcript, small nucleolar RNA, C/D Box 114 cluster in 2016 in the US (2). Currently, there is no effective approach for the detecting of EOC in early stage, because patients in early stage have no obvious symptoms. Almost $70 \%$ patients were diagnosed in advanced stage with predominant ascites and wide spread implant lesions in abdominal cavity. Ovarian cancer (OC) metastasis is characterized by the shedding of malignant cells from the surface of the ovary and their implantation onto the peritoneal surface $(3,4)$. Unlike other solid tumors, no anatomical barrier exists to block OC metastasis inside the peritoneal cavity. Although the metastasis of EOC has no common patterns, some researchers thought the omentum was the preferentially implanted site of EOC (5). The omentum, which is the largest peritoneal fold in the peritoneal cavity, plays critical role in several physiological process including fat deposition, immune contribution and infection isolation. The 'omental caking', indicating the thickness of omentum after invasion of $\mathrm{OC}$ at the late stage, is one of a distinct pattern of advanced EOC (6). In the relative early stage of EOC, the pathologist can also find $\mathrm{OC}$ cells in the omentum after surgery. Patients without omental caking show slighter metastasis and better prognosis (7). Thus, it was inferred that the omentum might actually provide 'optimal' sign for the migration of cancer cells. Therefore, we can know the metastatic manner of EOC by comparing the invaded omentum with the primary tumor. And it can provide novel potential biomarkers for screening the early metastasis and developing new treatment strategies of the treatment by resisting the spread of the cancer cells.

In the present study, we identified the non-coding RNAs (ncRNAs) between primary ovarian tumors and omental metastatic tissue using microarray analysis. The candidate altered genes, which selected by the microarray analysis results, were further validated by Reverse transcription quantitative polymerase chain reaction (RT-qPCR). We also clustered the subgroup based on patient age, International Federation of Gynecology and Obstetrics (FIGO) stage (8), pathological grade, and omental metastatic stage.

\section{Materials and methods}

Study samples. All the samples were collected after surgery performed in Xiangya Hospital of Central South University 
(Changsha, China) between March 2013 and May 2015. A total of 48 paired $\mathrm{OC}$ and omental fresh samples were stored in $-80^{\circ} \mathrm{C}$ for RT-qPCR analysis. The clinic pathologic data were obtained from medical records and pathologic reports. Prior informed consent was obtained from all recruited patients for ovarian carcinoma tissues collection and the publication of their data. The study was approved by the Ethics Committee of Xiangya Hospital of Central South University (reference: 201604556).

Gene chip hybridization and data analysis. Total RNA was extracted using the TRIzol method (Shanghai Sangong Pharmaceutical Co., Ltd., Shanghai, China) according to the manufacturer's instructions. Total RNA was quantified by the Nano Drop ND-2000 (Thermo Fisher Scientific, Inc., Waltham, MA, USA) and the RNA integrity was assessed using Agilent Bioanalyzer 2100 (Agilent Technologies, Inc., Santa Clara, CA, USA). Prime Script RT-PCR kit (Takara, Dalian, China) was utilized for reverse transcription, then total RNA were transcribed to double strand cDNA, after that synthesized into cRNA and labeled with Cyanine-3-CTP. The labeled cRNAs were hybridized onto the microarray. After washing, the arrays were scanned with an Agilent Scanner G2505C (Agilent Technologies, Inc.). Feature Extraction software (version 10.7.1.1; Agilent Technologies, Inc.) was used to obtain raw data from the array images. GeneSpring was employed for basic analysis of raw data. First, raw data were normalized with the quantile algorithm. Probes with at least 1 of 2 conditions having flags in ' $P$ ' were chosen for further data analysis. The ncRNAs were then identified through fold change as well as P-value calculated with t-test. The threshold set for up- and downregulated genes was a fold change $\geqq 2.0$ and a P-value $\leqq 0.05$. Finally, hierarchical clustering of genes based on clinic pathologic data was performed.

Quantitative real-time polymerase chain reaction. Total RNA was extracted using the TRIzol method and reverse transcribed to cDNA by using PrimeScript RT-PCR kit (Takara) in accordance with the manufacturer's protocol. Real time PCR was performed on a ViiA 7 Real-Time Fluorescent Quantitative PCR system (Thermo Fisher Scientific, Inc.). The PCR amplification conditions were 40 cycles of $95^{\circ} \mathrm{C}$ for $30 \mathrm{sec}, 95^{\circ} \mathrm{C}$ for $5 \mathrm{sec}, 60^{\circ} \mathrm{C}$ for $34 \mathrm{sec}$. Primers of target genes are as follows: U6 forward, 5'-CTCGCTTCGGCAGCACA-3' and reverse, 5'-AACGCTTCACGAATTTGCGT-3'. U6 was used as the reference gene. myocardial infarction associated transcript (MIAT) forward, 5'-GGAACAAGGATGGGAGTCG-3' and reverse, 5'-GCACTGAGCAAATGGAGACA-3'. Small nucleolar RNA, C/D Box 114 cluster (SNORD114)-10, reverse transcription primer, 5'-CTCAACTGGTGTCGTGGAGTC GGCAATTCAGTTGAGTGGACCTC-3', forward, 5'-ACA CTCCAGCTGGGAAGATCAATGATGACT-3' and universal primer, 5'-ACTGACTGATGCAATCTCAACTGGTGTCGT GGA-3'. SNORD114-2 reverse transcription primer, 5'-CTC AACTGGTGTCGTGGAGTCGGCAATTCAGTTGAGTGGA CCTC-3', forward, 5'-ACACTCCAGCTGGGGGACCAA TGATAATG-3' and universal primer, 5'-ACTGACTGATGC AATCTCAACTGGTGTCGTGGA-3'. SNORD114-11, reverse transcription primer, 5'-CTCAACTGGTGTCGTGGAGT CGGCAATTCAGTTGAGTGGACCTC-3', forward, 5'-ACA CTCCAGCTGGGTGGACCAGTGATGGTG-3' and universal primer, 5'-ACTGACTGATGCAATCTCAACTGGTGTCGT GGA-3'. Abundance was calculated using the 2- $\triangle \mathrm{CT}$ method. SPSS 18.0 statistical software package (SPSS, Inc., Chicago, IL, USA) was used for statistical analysis; error bars represent the standard error of the mean. Statistical analyses were performed using paired and t-test and non-parametric test. $\mathrm{P}<0.05$ was considered as statistically significant.

\section{Results}

Comprehensive analysis of ncRNAs in primary and metastatic $O C$. The differentiate expressed ncRNAs in 3 paired OC/omental metastasis (OM) samples were determined by microarray analysis. Hierarchical clustering revealed that 179 and 56 of the 235 differentially expressed ncRNAs were up- and downregulated, respectively (Fig. 1A). Among the ncRNAs, MIAT $(\mathrm{P}=0.015)$ was upregulated, whereas SNORD114-2 ( $\mathrm{P}=0.024)$ SNORD114-10 ( $\mathrm{P}=0.0002)$ and SNORD114-11 ( $\mathrm{P}=0.0001)$ were downregulated (Fig. 1B). We further validated the expression level of the four genes in 3 matched primary and metastatic OC by RT-qPCR, the expression trend of the genes were consistent with the results of chip (Fig. 2). MIAT ( $\mathrm{P}=0.0363$ ) expression increased in the omentum, while SNORD114-2 ( $\mathrm{P}=0.0246)$, SNORD114-10 $(\mathrm{P}=0.0025)$ and $\mathrm{SNORD114}-11(\mathrm{P}=0.1125)$ were downregulated in $\mathrm{OM}$ tissues compared to $\mathrm{OC}$ tissues.

The expressions of MIAT SNORD114-10 SNORD114-2 SNORD114-11 genes in OC. To validate the array data, RT-qPCR was performed with 27 pairs of ovarian and omentum cancer tissues. In the four genes, MIAT $(\mathrm{P}=0.023)$ was statistically significant upregulated in omentum tissues (Fig. 3A), while SNORD114-10 ( $\mathrm{P}=0.025)$ SNORD114-2 $(\mathrm{P}=0.082)$ and SNORD114-11 $(\mathrm{P}=0.132)$ were downregulated in omental tissues (Fig. 3B-D). Furthermore, to verify MIAT and SNORD114-10 expression changes indeed originate from cancer cells, we identified the MIAT and SNORD114-10expressionin another 21 early stage OC samples without OM compares with the expression in paired normal omental tissue. The expression level of these two genes showed no statistically significant difference between ovarian and omental tissues ( $\mathrm{P}>0.05)$ (Fig. 4A and B).

Association between genes expression and prognostic factors in $O C$. A total of 48 paired clinical samples analyzed above were derived from OC patients aged 21-77 years. The pathological appearance was divided into two stages as ovarian low-grade serous adenocarcinoma (LGSC) or high-grade serous adenocarcinoma (HGSC) according to the FIGO. The expression of SNORD114-10 in OC samples was not correlated with age, FIGO division, pathological classification or the presence of omental metastases $(\mathrm{P}>0.05)$ (Table I). This was essentially the same pattern as for MIAT, with the exception that levels of this ncRNA were significantly lower in omental samples with metastases $(\mathrm{P}=0.042)$ (Table I).

\section{Discussion}

The mortality rate of OC is ranked top one among all gynecological malignancies, and as high as $75 \%$ of diagnosed patients 
A

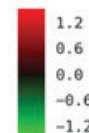

1.2
0.6
0.0
-0.6
-1.2

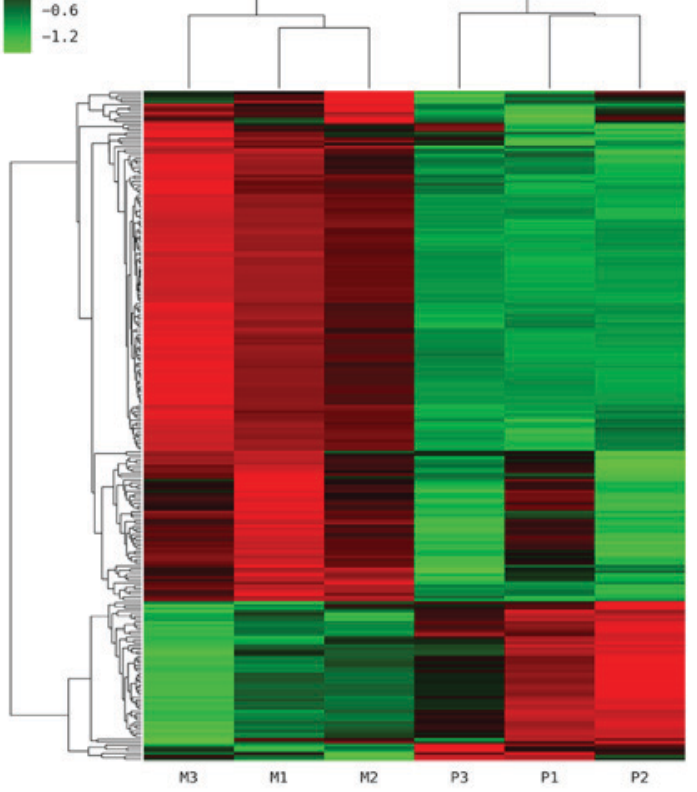

B

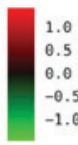

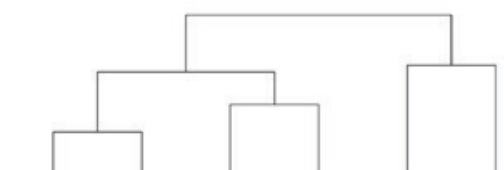

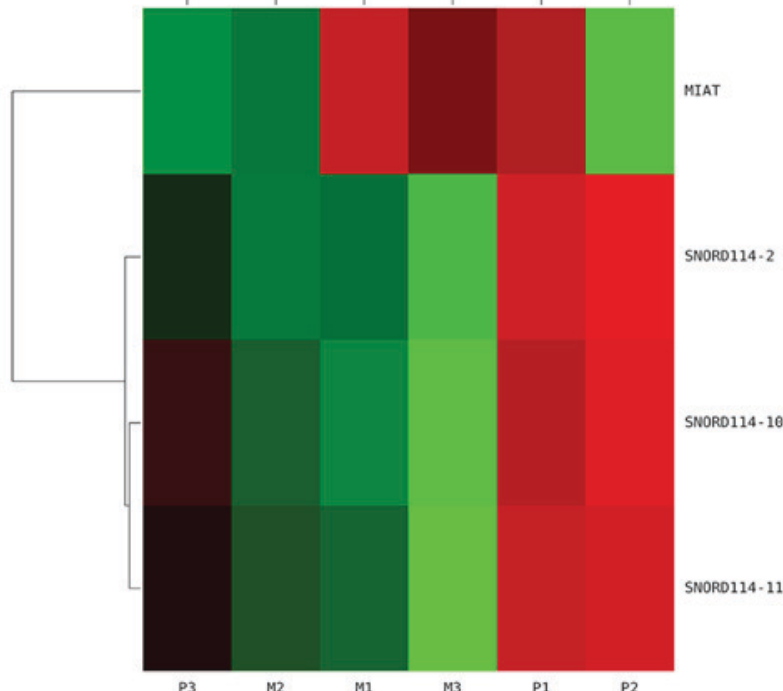

Figure 1. Hierarchical clustering map of ncRNAs. The heat map diagram shows the result of the two-way hierarchical clustering of ncRNAs and samples Each row represents a gene and each column represents a sample. P1, P2, P3 represent 3 cases of primary ovarian cancer sites, while M1, M2, M3 mean corresponding omental metastasis sites respectively. (A) A total of 235 ncRNAs were differentially expressed. (B) Of the significant ncRNAs expressed, MIAT $(\mathrm{P}=0.015)$ was upregulated in the OM tissues, whereas SNORD114-2 ( $\mathrm{P}=0.024)$, SNORD114-10 $(\mathrm{P}=0.0002)$ and SNORD114-11 ( $\mathrm{P}=0.0001)$ were downregulated in OM tissues compared with OC tissues. Red represents upregulated genes, and green represents downregulated genes. ncRNA, non-coding RNA; MIAT, myocardial infarction associated transcript; OM, omental metastasis; SNORD114, small nucleolar RNA, C/D Box 114 cluster; OC, ovarian cancer.
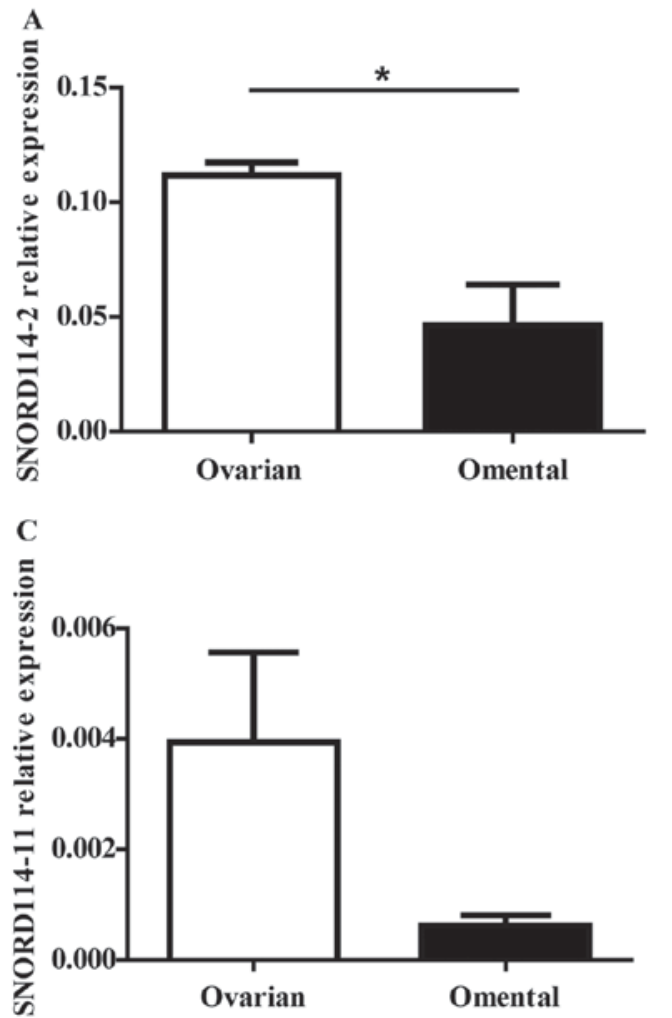

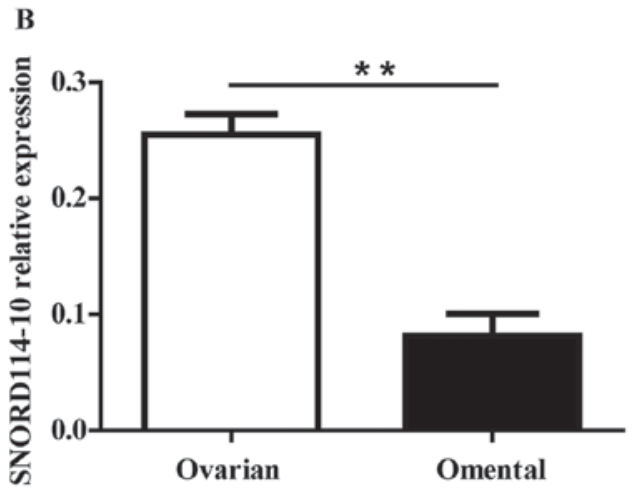

D

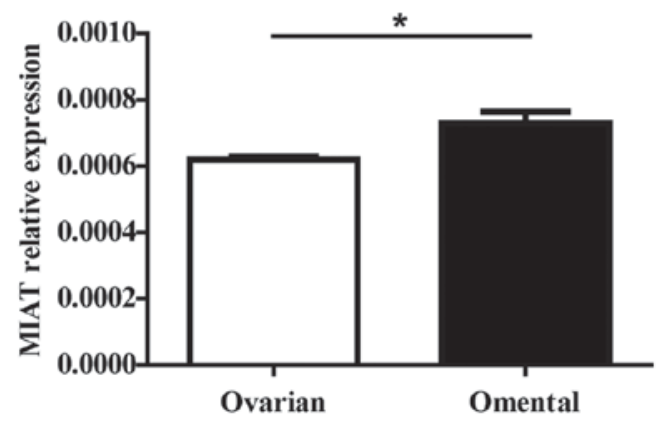

Figure 2. The significant expressed ncRNAs were selected for further validation by RT-qPCR. We further validated the expression level of the (A) SNORD114-2, (B) SNORD114-10 (C) SNORD114-11 and (D) MIAT genes in 3 matched primary and metastatic ovarian cancer by RT-qPCR, MIAT (P=0.0363) expression increased in the OM tissues while SNORD114-2 ( $\mathrm{P}=0.0246)$, SNORD114-10 ( $\mathrm{P}=0.0025)$ and SNORD114-11 $(\mathrm{P}=0.1125)$ were downregulated in OM tissues compared with OC tissues. The expression trend of the genes were consistent with the results of the chip. "P<0.05, ${ }^{* *} \mathrm{P}<0.01$. ncRNA, non-coding RNA; RT-qPCR, reverse transcription quantitative polymerase chain reaction; SNORD114, small nucleolar RNA, C/D Box 114 cluster; MIAT, myocardial infarction associated transcript; OM, omental metastasis; OC, ovarian cancer. 

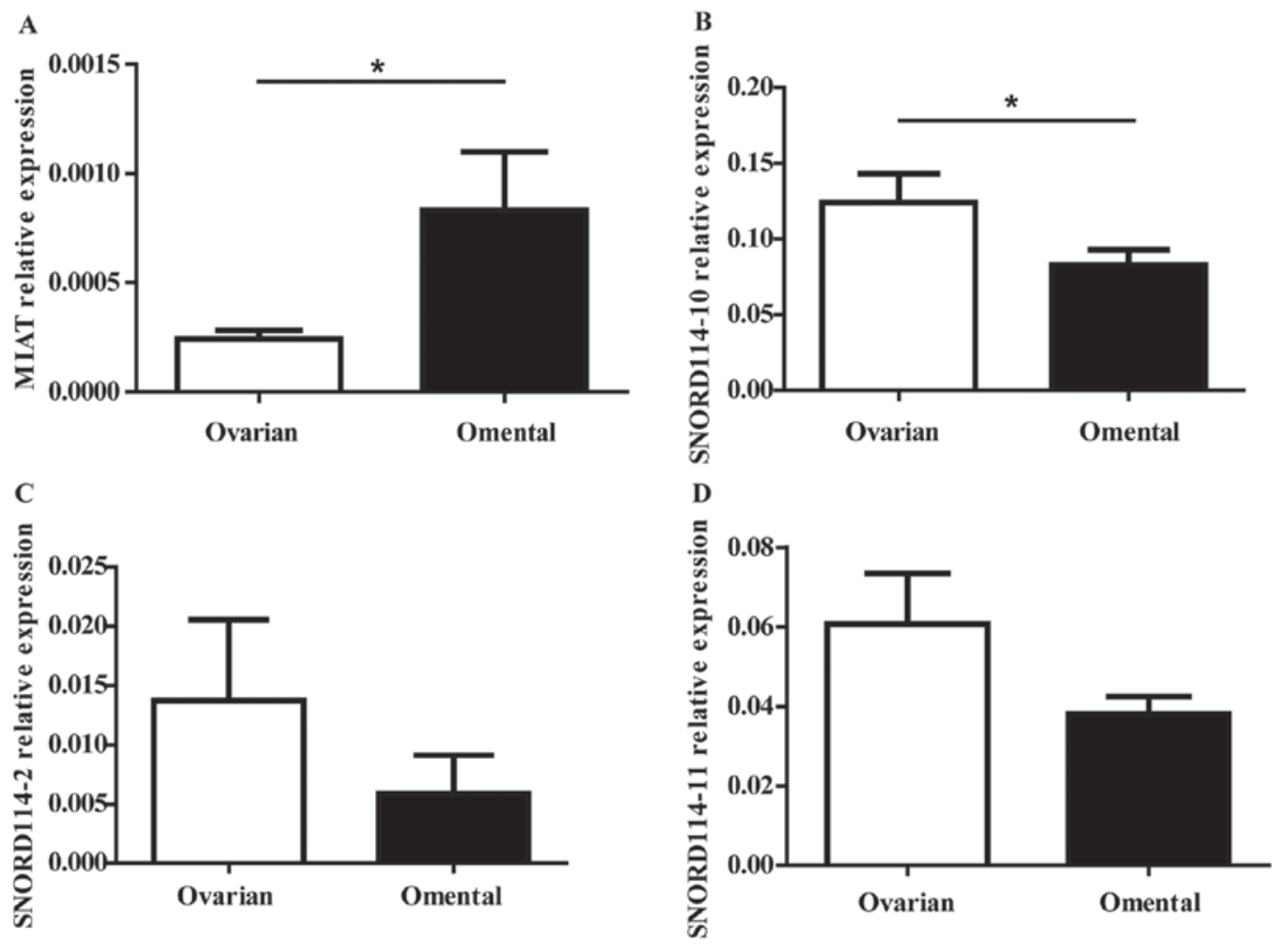

Figure 3. The expressions of MIAT, SNORD114-10, SNORD114-2 and SNORD114-11 in ovarian cancer and omental metastasis. Representative expression of the non-coding RNA by RT-qPCR in 27 pairs samples. (A) MIAT ( $\mathrm{P}=0.023)$ was significantly upregulated in omental cancer tissues. (B) SNORD114-10 $(\mathrm{P}=0.025)$ was significantly downregulated in omental cancer tissues. (C) SNORD114-2 ( $\mathrm{P}=0.082)$ and (D) SNORD114-11 ( $\mathrm{P}=0.132)$ were downregulated but they were not statistically significant. "P<0.05. MIAT, myocardial infarction associated transcript; SNORD114, small nucleolar RNA, C/D Box 114 cluster; RT-qPCR, reverse transcription quantitative polymerase chain reaction.
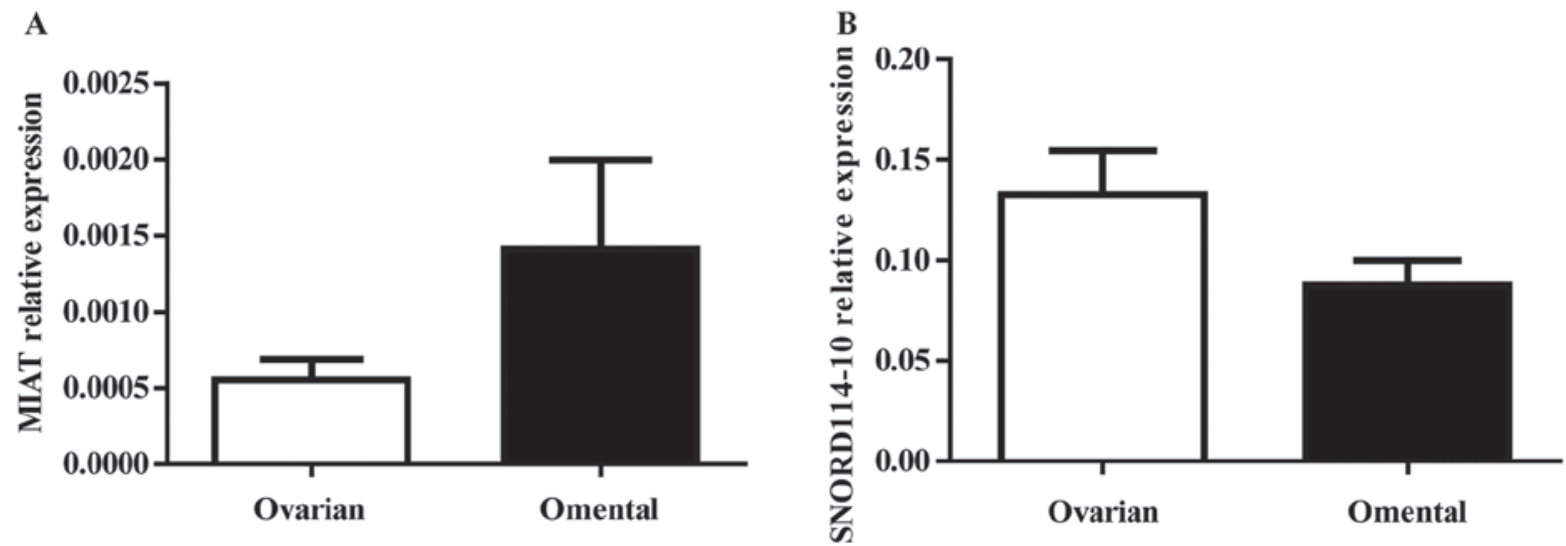

Figure 4. The expression level of MIAT and SNORD114-10 genes in ovarian cancer and normal omentum tissues. Representative expression of the non-coding RNA by RT-qPCR in 21 pairs samples which without omental metastasis. The expression level of the (A) MIAT (P=0.185) and (B) SNORD114-10 (P=0.134) showed no statistically significant difference between ovarian and omental tissues $(\mathrm{P}>0.05)$. MIAT, myocardial infarction associated transcript; SNORD114, small nucleolar RNA, C/D Box 114 cluster; RT-qPCR, reverse transcription quantitative polymerase chain reaction.

are already in the late stage. It is difficult to detect omentum metastasis in OC patients due to the lacking of obvious symptoms and specific markers at early stage. At the same time, limited understanding about the mechanism of tumor formation also hinders the development of new treatments. The ncRNA as a new tumor marker, in the occurrence and development of tumors play an important role in the treatment of cancer for people to provide a new way of thinking.

In the present study we used microarray to determine the ncRNAs profile between OC and OM. To the best of our knowledge, we are the first research group to report the alterations in ncRNA expression in OC, and to show that SNORD114-10 levels are reduced in OM, while MIAT levels are increased when compared to OC tissue. Consequently, we believe that the expression of MIAT and SNORD114-10 are important factors that contribute to migration of ovarian tumor cells from the primary site to the omentum.

$\mathrm{OC}$ is associated with the highest mortality rates of all gynecologic malignancies (9). The metastatic potential of OC cells is influenced by a series of complex process, with 
Table I. Correlations between MIAT and SNORD114-10 expression and clinicopathlogical features.

\begin{tabular}{|c|c|c|c|c|c|}
\hline \multirow[b]{2}{*}{ Factors } & \multirow[b]{2}{*}{ Cases } & \multicolumn{2}{|c|}{ MIAT } & \multicolumn{2}{|c|}{ SNORD114-10 } \\
\hline & & $2-\Delta \mathrm{CT}($ mean $\pm \mathrm{SD})$ & P-value & $2-^{\Delta \mathrm{CT}}($ mean $\pm \mathrm{SD})$ & P-value \\
\hline Age (years) & & & 0.597 & & 0.205 \\
\hline$<50$ & 22 & $0.00034 \pm 0.00035$ & & $0.1079 \pm 0.0816$ & \\
\hline$\geq 50$ & 26 & $0.00041 \pm 0.00055$ & & $0.1446 \pm 0.1108$ & \\
\hline FIGO stage & & & 0.251 & & 0.539 \\
\hline I-II & 13 & $0.00051 \pm 0.00039$ & & $0.1424 \pm 0.1078$ & \\
\hline III-IV & 35 & $0.00033 \pm 0.00049$ & & $0.1224 \pm 0.0969$ & \\
\hline FIGO grade & & & 0.434 & & 0.100 \\
\hline Low & 9 & $0.00049 \pm 0.00033$ & & $0.0787 \pm 0.068$ & \\
\hline High & 39 & $0.00038 \pm 0.00031$ & & $0.1391 \pm 0.1024$ & \\
\hline Omentum metastasis & & & $0.042^{\mathrm{a}}$ & & 0.773 \\
\hline Yes & 27 & $0.00024 \pm 0.00020$ & & $0.1241 \pm 0.0999$ & \\
\hline No & 21 & $0.00055 \pm 0.00063$ & & $0.1326 \pm 0.1005$ & \\
\hline
\end{tabular}

${ }^{a} \mathrm{P}<0.05$. MIAT, myocardial infarction associated transcript; SNORD114, small nucleolar RNA, C/D Box 114 cluster; FIGO, International federation of Gynecology and Obstetrics; SD, standard deviation.

the most common metastatic sites being the peritoneum and omentum and the disease progresses rapidly when malignant cells reach these secondary sites (4). Based on the 'seed and soil' hypothesis, we decided to investigate genes that may contribute to the 'soil' properties of the omentum.

According to the results of RT-PCR, MIAT was upregulated and SNORD114-10 was downregulated in the omentum in metastatic disease cases. However, there was no statistical difference between $\mathrm{OC}$ with $\mathrm{OM}$ and $\mathrm{OC}$ without OM. Thus, we speculate that the migration in $\mathrm{OC}$ is not a tumor cell-intrinsic property, but rather is dependent on the omentum 'soil' which provides the conditions for tumor development and subsequent disease acceleration.

ncRNAs usually perform catalytic or regulatory functions (10), and can be classified as small (<200 bps) or long ncRNAs (>200 bps) (11). Over the past few years, many ncRNAs have been identified including H19(12) and Xist (13). Our present study demonstrates that the expression of SNORD114-10 and MIAT were differentially regulated in metastatic omentum compared to non-infiltrated tissue. Previous studies have shown that some lncRNAs are differentially expressed in primary and metastatic tumors $(14,15)$. Metastasis-associated lung adenocarcinoma transcript 1 (MALAT1) is a well-known carcinogenic lncRNA that is highly expressed in lung metastases (16). Elevated MALAT1 in different tumors predicts shorter metastasis-free survival (MFS), deep tissue invasion (17), higher histological grade (18), and lower overall survival (OS) (19). Similar to MALAT1, HOX transcript antisense intergenic RNA (HOTAIR) is closely related to the occurrence and development of tumor metastasis (20-23). Zhang et al (24) found that MIR4697 host gene (MIR4697HG) in cancerous tissues increased compared with that in adjacent noncancerous tissues. It could promote OC growth and metastasis. The aggressive role of MIR4697HG in OC may be related to the ERK and AKT signaling pathways.
MIAT was described as a ncRNA expressed in mitotic progenitors (25), and RNA-FISH indicated that it was expressed in the inner nuclear layer (INL) and the ganglion cell layer (GCL) in humans. The research shows that MIAT functional abnormalities are associated with the risk of myocardial infarction, it also plays a regulatory role in other areas, including retinal cell growth, brain development and fibrosis after filamentous fibrosis (26,27). Moreover, a previous study reported that high expression of MIAT leads to abnormal endothelial cell proliferation and cell migration during early retinal microvascular dysfunction (28). Luan et al (29) found that MIAT downregulation inhibited the epithelial-mesenchymal transition (EMT) and decreased migration and invasion in MDA-MB-231 and MCF-7 breast cancer cell lines. They also suggested that MIAT promotes breast cancer progression and functions as competing endogenous RNA (ceRNA) to regulate dual specificity phosphatase 7 (DUSP7) expression by sponging miR-155-5p in breast cancer. Shen et al (30) found that MIAT acted as a ceRNA, and formed a feedback loop with AKT and miR-150-5p to regulate Human lens epithelial cell (HLEC) function. Our current results showing that MIAT was almost 3-fold higher in OM tissue when compared with OC. Therefore, we hypothesize that MIAT may promote the metastasis of OC, which may be through the regulation of certain genes or through the EMT or AKT pathway to promote OC metastasis. Next we will determine whether MIAT can regulate migration in OC cells by silencing and over-expressing MIAT. Further study are required to determine the role of MIAT (the correlation between) in OC metastasis/the metastssis of OC.

Emerging evidence indicates that snoRNAs can play several non-classical roles, including reactive oxygen species scavenging in the cytoplasm and being precursors for microRNA-like molecules (31-33). Although the functional map of snoRNA has not been fully elucidated, snoRNA is likely to play an important regulatory role in the development 
and progression of cancer. According to new molecular mechanisms that have been found (34). Some snoRNAs are implicated in tumorigenesis, such as the tumor suppressors SNOR12 and SNOR44, and the tumor promoters SNOD33 and U70C (35). Interestingly, Mei et al reported high expression of SNORA42 in lung cancer, and concluded that this ncRNA might be oncogenic (36). Gee et al (37) demonstrated that SNORD48 expression was significantly lower in low-grade breast cancers, whereas high SNORD44 expression in breast and non-small cell lung cancer indicated a better prognosis. Therefore, SNORD44 and SNORD48 play a suppressing tumor function role in these cancers. Valleron et al found SNORD112, SNORD113 and SNORD114 ectopic expression in acute promyelocytic leukemia (APL), and further molecular studies also shown SNORD114-1 variants through the retinoblastoma gene $(\mathrm{Rb}) / \mathrm{p} 16$ signaling pathway, allowing cells to block $\mathrm{G} 0 / \mathrm{G} 1$ and reduce the proportion of $\mathrm{S}$ phase, thereby inhibiting cell growth (38). At the same time, it has been found that SNORD123 was highly expressed in colorectal cancer cells and could affect tumorigenesis by epigenetic modification (39). The levels of SNORD33, SNORD66 and SNORD76 in the plasma of patients with non-small cell lung cancer were significantly higher than those in healthy and chronic obstructive pulmonary diseases, and had the value of early diagnosis of lung cancer (40). Overall, snoRNA was closely correlated with the pathogenesis and progression of various tumors, but the expression pattern and functional role of snoRNA in OC has not been elucidated.

Here, we found that SNORD114-10 levels were reduced in metastatic omentum, suggesting that this snoRNA might have properties that suppress migration of ovarian tumor cells to secondary sites. SNORD114-10 maybe inhibited the metastasis of OC by regulating the expression of certain genes or through the EMT or AKT pathway. Next we will determine whether SNORD114-10 can regulate migration in OC cells by silencing and over-expressing SNORD114-10. Further study are required to determine the role of SNORD114-10 (the correlation between) in OC metastasis/the metastssis of OC.

Because preoperative OC omentum metastasis is difficult to diagnose, so it is not conducive to preoperative surgical options. Therefore, we believe that MIAT and SNORD114-10 can be used as a marker of OC OM, which can guide OC patients' preoperative choice.

\section{Acknowledgements}

The present study was supported by the Fundamental Research Funds of Central South University (no. 2015zzts297), Program for Science and Technology Plan of Hunan Province (no. 2013FJ4114) and Applied of Fundamental Research Key Prjoect (no. 2016JC2036). We are grateful for all the contributions that supported this study.

\section{References}

1. Liu CM: Cancer of the ovary. N Engl J Med 352: 1268-1269, 2005.

2. Siegel RL, Miller KD and Jemal A: Cancer Statistics, 2017. CA Cancer J Clin 67: 7-30, 2017.

3. Lengyel E: Ovarian cancer development and metastasis. Am J Pathol 177: 1053-1064, 2010.
4. Naora $\mathrm{H}$ and Montell DJ: Ovarian cancer metastasis: Integrating insights from disparate model organisms. Nat Rev Cancer 5: 355-366, 2005

5. PradeepS,KimSW,WuSY,Nishimura M,Chaluvally-RaghavanP, Miyake T, Pecot CV, Kim SJ, Choi HJ, Bischoff FZ, et al: Hematogenous metastasis of ovarian cancer: Rethinking mode of spread. Cancer Cell 26: 77-91, 2014.

6. Cooper C, Jeffrey RB, Silverman PM, Federle MP and Chun GH: Computed tomography of omental pathology. J Comput Assist Tomogr 10: 62-66, 1986.

7. Liu T,Zhao L, Chen W, Li Z, Hou H, Ding L and Li X: Inactivation of von Hippel-Lindau increases ovarian cancer cell aggressiveness through the HIF1 $\alpha /$ miR-210/VMP1 signaling pathway. Int J Mol Med 33: 1236-1242, 2014.

8. Prat J; FIGO Committee on Gynecologic Oncology: Staging classification for cancer of the ovary, fallopian tube and peritoneum: Abridged republication of guidelines from the international federation of gynecology and obstetrics (FIGO). Obstet Gynecol 126: 171-174, 2015.

9. Torre LA, Bray F, Siegel RL, Ferlay J, Lortet-Tieulent J and Jemal A: Global cancer statistics, 2012. CA Cancer J Clin 65: 87-108, 2015.

10. Carninci $P$ and Hayashizaki Y: Noncoding RNA transcription beyond annotated genes. Curr Opin Genet Dev 17: 139-144, 2007.

11. Pauli A, Rinn JL and Schier AF: Non-coding RNAs as regulators of embryogenesis. Nat Rev Genet 12: 136-149, 2011.

12. Keniry A, Oxley D, Monnier P, Kyba M, Dandolo L, Smits G and Reik W: The H19 lincRNA is a developmental reservoir of miR-675 that suppresses growth and Igf1r. Nat Cell Biol 14: 659-665, 2012.

13. McHugh CA, Chen CK, Chow A, Surka CF, Tran C, McDonel P, Pandya-Jones A, Blanco M, Burghard C, Moradian A, et al: The Xist lncRNA interacts directly with SHARP to silence transcription through HDAC3. Nature 521: 232-236, 2015.

14. Tahira AC, Kubrusly MS, Faria MF, Dazzani B, Fonseca RS, Maracaja-Coutinho V, Verjovski-Almeida S, Machado MC and Reis EM: Long noncoding intronic RNAs are differentially expressed in primary and metastatic pancreatic cancer. Mol Cancer 10: 141, 2011.

15. Matouk IJ, Abbasi I, Hochberg A, Galun E, Dweik H and Akkawi M: Highly upregulated in liver cancer noncoding RNA is overexpressed in hepatic colorectal metastasis. Eur J Gastroenterol Hepatol 21: 688-692, 2009.

16. Ji P, Diederichs S, Wang W, Böing S, Metzger R, Schneider PM, Tidow N, Brandt B, Buerger H, Bulk E, et al: MALAT-1, a novel noncoding RNA and thymosin beta4 predict metastasis and survival in early-stage non-small cell lung cancer. Oncogene 22: 8031-8041, 2003.

17. Han Y, Liu Y, Nie L, Gui Y and Cai Z: Inducing cell proliferation inhibition, apoptosis and motility reduction by silencing long noncoding ribonucleic acid metastasis-associated lung adenocarcinoma transcript 1 in urothelial carcinoma of the bladder. Urology 81: 209.e1-e7, 2013.

18. Hou Z, Xu X, Zhou L, Fu X, Tao S, Zhou J, Tan D and Liu S: The long non-coding RNA MALAT1 promotes the migration and invasion of hepatocellular carcinoma by sponging miR-204 and releasing SIRT1. Tumour Biol 39: 1010428317718135, 2017.

19. Schmidt LH, Spieker T, Koschmieder S, Schäffers S, Humberg J, Jungen D, Bulk E, Hascher A, Wittmer D, Marra A, et al: The long noncoding MALAT-1 RNA indicates a poor prognosis in non-small cell lung cancer and induces migration and tumor growth. J Thorac Oncol 6: 1984-1992, 2011.

20. Gupta RA, Shah N, Wang KC, Kim J, Horlings HM, Wong DJ, Tsai MC, Hung T, Argani P, Rinn JL, et al: Long non-coding RNA HOTAIR reprograms chromatin state to promote cancer metastasis. Nature 464: 1071-1076, 2010.

21. Xu ZY, Yu QM, Du YA, Yang LT, Dong RZ, Huang L, Yu PF and Cheng XD: Knockdown of long non-coding RNA HOTAIR suppresses tumor invasion and reverses epithelial-mesenchymal transition in gastric cancer. Int J Biol Sci 9: 587-597, 2013.

22. Nakagawa T, Endo H, Yokoyama M, Abe J, Tamai K, Tanaka N, Sato I, Takahashi S, Kondo T and Satoh K: Large noncoding RNA HOTAIR enhances aggressive biological behavior and is associated with short disease-free survival in human non-small cell lung cancer. Biochem Biophys Res Commun 436: 319-324, 2013.

23. Li HM, Yang H, Wen DY, Luo YH, Liang CY, DH, Ma W, Chen G, $\mathrm{He} \mathrm{Y}$ and Chen JQ: Overexpression of LncRNA HOTAIR is associated with poor prognosis in thyroid carcinoma: A study based on TCGA and GEO data. Horm Metab Res 49: 388-399, 2017. 
24. Zhang LQ, Yang SQ, Wang Y, Fang Q, Chen XJ, Lu HS and Zhao LP: Long noncoding RNA MIR4697HG promotes cell growth and metastasis in human ovarian cancer. Anal Cell Pathol (Amst) 2017: 8267863, 2017.

25. Tsuiji H, Yoshimoto R, Hasegawa Y, Furuno M, Yoshida M and Nakagawa S: Competition between a noncoding exon and introns: Gomafu contains tandem UACUAAC repeats and associates with splicing factor-1. Genes Cells 16: 479-90, 2011.

26. Mercer TR, Dinger ME, Sunkin SM, Mehler MF and Mattick JS Specific expression of long noncoding RNAs in the mouse brain. Proc Natl Acad Sci USA 105: 716-721, 2008.

27. Sone M, Hayashi T, Tarui H, Agata K, Takeichi $M$ and Nakagawa S: The mRNA-like noncoding RNA gomafu constitutes a novel nuclear domain in a subset of neurons. J Cell Sci 120: 2498-2506, 2007.

28. Yan B, Yao J, Liu JY, Li XM, Wang XQ, Li YJ, Tao ZF, Song YC, Chen Q and Jiang Q: IncRNA-MIAT regulates microvascular dysfunction by functioning as a competing endogenous RNA. Circ Res 116: 1143-1156, 2015.

29. Luan T, Zhang X, Wang S, Song Y, Zhou S, Lin J, An W, Yuan W, Yang Y, Cail H, et al: Long non-coding RNA MIAT promotes breast cancer progression and functions as ceRNA to regulate DUSP7 expression by sponging miR-155-5p. Oncotarget 8: 76153-76164, 2017.

30. Shen Y, Dong LF, Zhou RM, Yao J, Song YC, Yang H, Jiang Q and Yan B: Role of long non-coding RNA MIAT in proliferation, apoptosis and migration of lens epithelial cells: A clinical and in vitro study. J Cell Mol Med 20: 537-548, 2016.

31. Scott MS and Ono M: From snoRNA to miRNA: Dual function regulatory non-coding RNAs. Biochimie 93: 1987-1992, 2011.

32. Brameier M, Herwig A, Reinhardt R, Walter L and Gruber J: Human box C/D snoRNAs with miRNA like functions: Expanding the range of regulatory RNAs. Nucleic Acids Res 39: 675-686, 2011
33. Ono M, Scott MS, Yamada K, Avolio F, Barton GJ and Lamond AI: Identification of human miRNA precursors that resemble box C/D snoRNAs. Nucleic Acids Res 39: 3879-3891, 2011.

34. Mannoor K, Liao J and Jiang F: Small nucleolar RNAs in cancer. Biochim Biophys Acta 1826: 121-128, 2012.

35. Thorenoor N and Slaby O: Small nucleolar RNAs functioning and potential roles in cancer. Tumour Biol 36: 41-53, 2015.

36. Mei YP, Liao JP, Shen J, Yu L, Liu BL, Liu L, Li RY, Ji L, Dorsey SG, Jiang ZR, et al: Small nucleolar RNA 42 acts as an oncogene in lung tumorigenesis. Oncogene 31: 2794-2804, 2012.

37. Gee HE, Buffa FM, Camps C, Ramachandran A, Leek R, Taylor M, Patil M, Sheldon H, Betts G, Homer J, et al: The small-nucleolar RNAs commonly used for microRNA normalisation correlate with tumour pathology and prognosis. $\mathrm{Br} \mathrm{J}$ Cancer 104: 1168-1177, 2011.

38. Valleron W, Laprevotte E, Gautier EF, Quelen C, Demur C, Delabesse E, Agirre X, Prósper F, Kiss T and Brousset P: Specific small nucleolar RNA expression profiles in acute leukemia. Leukemia 26: 2052-2060, 2012.

39. Ferreira HJ, Heyn H, Moutinho $\mathrm{C}$ and Esteller M: CpG island hypermethylation-associated silencing of small nucleolar RNAs in human cancer. RNA Biol 9: 881-890, 2012.

40. Liao J, Yu L, Mei Y, Guarnera M, Shen J, Li R, Liu Z and Jiang F: Small nucleolar RNA signatures as biomarkers for non-small-cell lung cancer. Mol Cancer 9: 198, 2010.

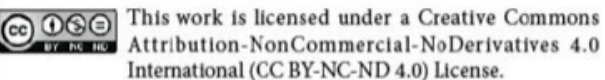

